\title{
An Evaluation of Methods for Neuromagnetic Image Reconstruction
}

\author{
BRIAN JEFFS, RICHARD LEAHY, MEMBER, IEEE, AND MANBIR SINGH, MEMBER, IEEE
}

\begin{abstract}
In this paper, we discuss several aspects of a potential new medical imaging modality for producing a quantitative three-dimensional map of neuron current densities associated with brain function. The neuromagnetic image is produced by reconstructing a current dipole field from external magnetic field measurements made with an array of superconducting quantum interference device (SQUID) detectors. This field is produced by numerical inversion of the Biot-Savart equation. The purpose of the work is to investigate fundamental limits on the feasibility of the proposed system under ideal conditions. The following problems are addressed: 1) What are the factors limiting resolution of the system? 2) What is a suitable model for neural activity in the brain? 3) What classes of algorithms are suitable for estimating the model parameters? The major conclusion of this work is that the inversion problem is severely ill-posed and the choice of model and estimation algorithm are crucial in obtaining reasonable solutions. $A$ class of solutions, termed minimum dipole, is proposed as a means of obtaining more acceptable results.
\end{abstract}

\section{I. "Neuromagnetic Imaging"}

$\mathrm{T}$ HE aim of neuromagnetic imaging (NMI) is to produce maps of neuron current densities resulting from brain activity associated with both evoked response to external stimuli and hopefully the higher thought processes. Neuromagnetic images are produced by reconstructing the neuron current field from measurements of the induced external magnetic field. This imaging technique differs from the magnetoencephalogram (MEG) isofield mappings of the skull [1] since the internal brain electrical activity, rather than the external magnetic field, is displayed. The MEG maps are obtained directly from the data, whereas the NMI field requires the solution of an ill-posed inverse problem. As with the MEG maps, we treat NMI as a magnetostatic problem by sampling the time series of the neuromagnetic waveforms from the detector at a single time of interest so as to isolate the response component we wish to analyze. It is our intention to address the feasibility and fundamental limitations of

Manuscript received Ocober 24, 1986; revised February 23, 1987. This work was supported in part by the Hughes Aircraft Company Doctoral Fellowship Program and also in part by the USC Faculty Research and Innovation Fund.

B. Jeffs is with Hughes Aircraft Company, Buena Park, CA 90621 and the Department of Electrical Engineering-Systems, Signal and Image Processing Institute, University of Southern California, Los Angeles, CA 90089.

R. Leahy is with the Department of Electrical Engineering-Systems, Signal and Image Processing Institute, University of Southern California, Los Angeles, CA 90089.

M. Singh is with the Department of Radiology, University of Southern California, Los Angeles, CA 90033.

IEEE Log Number 8715576. this imaging modality and to evaluate the effectiveness of several reconstruction algorithms commonly employed in medical image reconstruction.

Magnetic fields associated with evoked responses may be measured on a sample grid around the skull using a SQUID biomagnetometer [1], [2], [3]. These data have been used to estimate the location, orientation, and magnitude of a single current dipole in the brain which best fits the data in a least squares sense [1], [4], [5]. This approach is useful when the current field of interest is known to be localized to a single small region of the brain. Neuromagnetic imaging extends this concept to the case of multiple-current dipole sources or more complex distributed current fields which would exist when separated regions of the brain are involved in a response, or during higher brain functions. In these cases, the single-dipole model is a poor match [5], [6] and we are motivated to treat the problem as one of image reconstruction. Our approach then emphasizes solutions to the inverse problem (current field from magnetic measurements) using image reconstruction algorithms and techniques which one might encounter in computed tomography or other medical imaging applications.

Neuromagnetic imaging on a single reconstruction plane in the brain was first demonstrated by Singh et al. [7]. Their approach used discrete samples of the evoked magnetic field to reconstruct a current field modeled as discrete current dipole cells all lying in a single plane with either one- or two-constrained dipole orientations. The depth of this single plane was adjusted for best fit with the measured data. We propose an extension to this model which will include three-dimensional reconstructed images and multiple-dipole orientations. This allows the estimation of dipole distributions in depth as well as lateral position and orientation.

The paper is organized as follows. In Section II, we introduce the basic physical model we will use for NMI and develop the mathematical formulation of this model. We then discuss possible constraints on the solution and how these may be included in the formulation. In Section III, the fundamental limits on image resolution due to background noise, the SQUID response, and the ill-conditioned nature of the system matrix are discussed. In Section IV, we present several algorithms which can be applied to the NMI reconstruction problem and evaluate their effectiveness. Results of computer model evaluation of these algorithms are also included. 


\section{Models for Neuromagnetic IMAging}

\section{A. Basic Formulation}

For NMI reconstruction, we adopt the simple physical model of Fig. 1. A spheroid shaped reconstruction volume represents the interior of the skull which will contain a 3-D distribution of neuron currents. Measurements of the external magnetic field are taken at points on the sampling surface represented by the hemispherical shell. These points correspond to the positions on the skull where measurements with the SQUID gradiometer are taken. Our goal is to infer the current distribution from these measurements.

The relationship between a continuous vector current field and its induced magnetic field at a point $r$ in space is given by the vector integral form of the Biot-Savart Law

$$
B(\underline{r})=\frac{\mu_{0}}{4 \pi} \int \frac{J\left(\underline{r}^{\prime}\right) \times\left(\underline{r}-\underline{r}^{\prime}\right)}{\left|\underline{r}-\underline{r}^{\prime}\right|^{3}} d^{3} \underline{r}^{\prime}
$$

where $J\left(\underline{r}^{\prime}\right)$ denotes the vector current density at $\underline{r}^{\prime}$ and $\mu$ the magnetic permiability of the medium, which we approximate with $\mu_{0}$, the permiability of free space. Although the brain's current field consists of discrete firings of individual neuron cells, the high density of neurons in brain tissue and the inability of present instrumentation to resolve single cell current flow make this continuous field model an accurate one. However, we can take only a finite number of magnetic field measurements, and in order to reduce the dimensionality of the problem and express this nonlinear relationship as a set of linear equations we approximate (1) with a discrete sum. The vector current field is replaced by a finite number of current dipoles, $Q\left(\underline{r}_{i}\right)$ located in a three-dimensional grid where a dipole's orientation and magnitude are determined by integrating the current field over the volume cell (voxel) surrounding the dipole. Equation (1) becomes

$$
B\left(\underline{r}_{m}\right)=\frac{\mu_{0}}{4 \pi} \sum_{n=1}^{N} \frac{Q\left(\underline{r}_{n}\right) \times\left(\underline{r}_{m}-\underline{r}_{n}\right)}{\left|\underline{r}_{m}-\underline{r}_{n}\right|^{3}}
$$

where $N$ is the total number of dipole cells and $m$ and $n$ are the indexes on the discrete sample points in space. This equation can be rewritten in vector-matrix form as the linear system

$$
\underline{B}=W \underline{Q}
$$

where

$$
\begin{aligned}
\underline{B}= & {\left[B_{x 1}, B_{y 1}, B_{z 1}, \cdots B_{x M}, B_{y M}, B_{z M}\right]^{T} } \\
B_{x m}= & x \text { component of } \underline{B}\left(\underline{r}_{m}\right) \\
\underline{Q}= & {\left[Q_{x 1}, Q_{y 1}, Q_{z 1}, \cdots, Q_{x N}, Q_{y N}, Q_{z N}\right]^{T} } \\
W= & {\left[\begin{array}{ccc}
W_{1,1} & \cdots & W_{1, N} \\
\vdots & & \vdots \\
W_{M, 1} & \cdots & W_{M, N}
\end{array}\right], } \\
& \text { a }(3 M \times 3 N) \text { block matrix }
\end{aligned}
$$

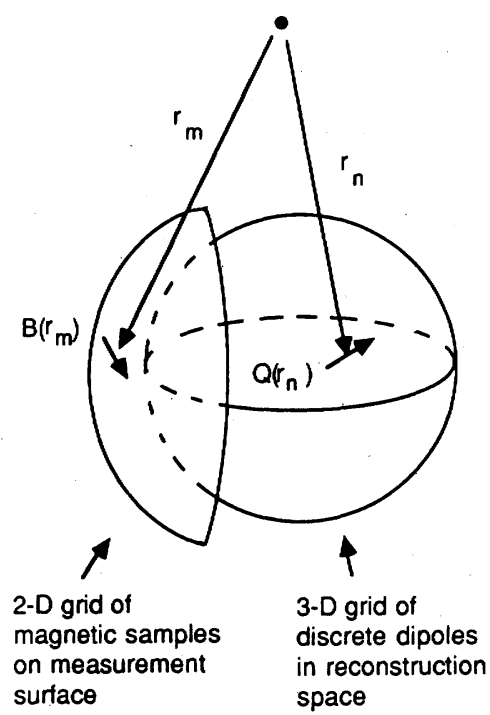

Fig. 1. Basic physical model for neuromagnetic imaging.

$$
\begin{aligned}
W_{m, n} & =\frac{\mu_{0}}{4 \pi\left|\underline{r}_{m}-\underline{r}_{n}\right|^{3}}\left[\begin{array}{ccc}
0 & r_{z: m, n} & -r_{y: m, n} \\
-r_{z: m, n} & 0 & r_{x: m, n} \\
r_{y: m, n} & -r_{x: m, n} & 0
\end{array}\right] \\
r_{x: m, n} & =x \text { component of }\left(\underline{r}_{m}-\underline{r}_{n}\right) .
\end{aligned}
$$

With the addition of an independent noise term to represent measurement error, the system is expressed in a form suitable for applying digital image reconstruction techniques, i.e., we may solve the inverse problem of finding $\underline{Q}$ given the measurement vector $\underline{B}$ where

$$
\underline{B}=W \underline{Q}+\underline{N} \text {. }
$$

The formulation of the entries in matrix $W$ can be adjusted to compensate for the fact that a SQUID gradiometer does not provide an exact measurement of the point flux density. Methods for doing so are discussed in Section III-B.

\section{B. Source Model and Constraints}

In practical applications of NMI, the system of (4) is highly underdetermined (see Section III) with many more degrees of freedom in the solution space that can be resolved by the sampled data. It is clear from this ambiguity that we cannot produce a meaningful reconstruction of a current dipole distribution without introducing a restrictive model based on the physiology of the brain. Any constraints imposed by this model can be used to reduce the dimensionality of the solution space and direct our selection from the infinite set of possible solutions to one that is useful. It should be stressed however that in using such a prior model to obtain reconstructions, the resulting images should be interpreted with the understanding that they are valid only to the extent that the model assumptions are correct.

It has been common to model the brain and skull as a nonconductive sphere or spheroid casing filled with a homogeneous conductive medium in which current dipole activity exists [1], [8]. Use of the spherical model enables 
the introduction of several simplifications and constraints. Volume currents which are induced in the conducting medium by a current dipole source do not contribute to the external magnetic field normal to the sphere surface, but may contribute to the tangential field components [1], [9]. Also, dipoles aligned with the sphere's radii, and radially symmetric dipole distributions produce no measurable external magnetic field [8]. The "invisibility" of these sources makes their inclusion in a reconstruction solution meaningless, so we may exclude such dipoles from our solution. The skull is not a perfect sphere, however, Barth et al. [10] have shown that field measurements of dipoles implanted in a human skull, even with gross cranial distortions, show little deviation from that predicted by a spherical model. Also, most areas of the skull can be fit to a spherical segment with a local radial center. These considerations enable us to utilize the constraints offered by the spherical conductor model even when our reconstruction space is not exactly spherical.

The brain region of primary interest for NMI is the cortex. Neurons within the cortex, and thus the current paths, are arranged predominantly normal to the local surface of the cortex [11]. Thus, given its shape, it is possible to constrain the orientation of the current dipoles which model the neuron activity. The cortex is highly convoluted [11], so if the orientation of the current dipole is to be constrained, the true shape of the cortex must be employed to estimate the direction. However, given the limited resolution of the system, we believe a model using the major features and landmarks of the cortex shape is sufficient for an initial study.

In [12], the shape of the cortex in slices of a human brain was digitized and stored as a brain atlas. Using various geometrical warping transforms, the shape could be adapted to different brain sizes with reasonable accuracy. Data of this kind could be employed in our model as an initial estimate of current dipole orientations near the major fissures and features of the brain. Individual brain geometry data for each patient could be obtained from magnetic resonance or X-ray computed tomography if more resolution is needed.

We propose the following simple model for the cortex which allows us to incorporate the above constraints (see Fig. 2). This model describes how the reconstruction volume of Fig. 1 is to be discretized. A series of horizontal slices represent planar slab sections through the brain; the cortex is then constrained to lie in a region spanned by concentric ellipses. By adjusting the major and minor axes of these ellipses it is possible to adapt the model to differing brain sizes. To digitize the problem, we segment each of the elliptical slabs into a set of voxels in each of which we allow a single equivalent current dipole. The spatial orientation of each dipole can be constrained to a specific direction. The number of voxels in each ring is reduced as distance from the skull surface increases. This reduction in resolution coincides with the loss of resolution at depth implied by the Biot-Savart law which is discussed in Section III-A.

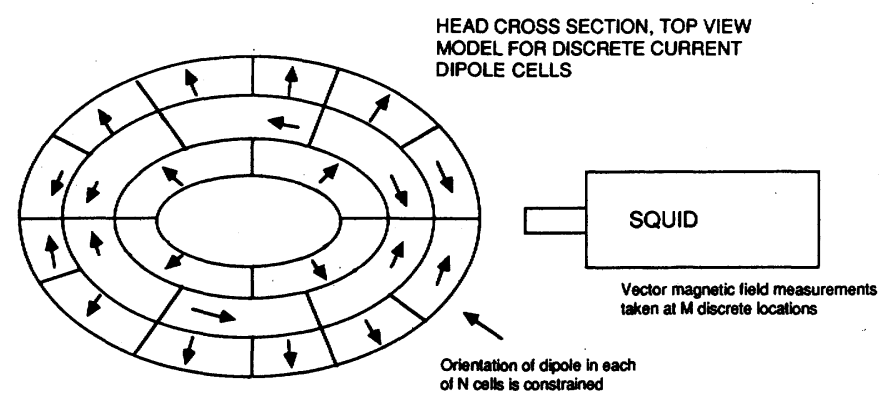

Fig. 2. Cortex sample grid and model showing constrained dipole orientation.

The above constraints are incorporated in the system by modifying (2) as follows:

$$
B\left(\underline{r}_{m}\right)=\frac{\mu_{0}}{4 \pi} \sum_{n=1}^{N} \frac{\left|Q_{n}\right| \underline{z}_{n}\left(\underline{r}_{n}\right) \times\left(\underline{r}_{m}-\underline{r}_{n}\right)}{\left|\underline{r}_{m}-\underline{r}_{n}\right|^{3}}
$$

where $\underline{z}_{n}\left(\underline{z}_{n}\right)$ denotes the unit vector representing the orientation of the $n$th current dipole of magnitude $\left|Q_{n}\right|$. Expressing this as a vector-matrix equation, we modify (4) as follows. Let $\underline{Q}=D \underline{Q}^{\prime}$ where $D$ is a tridiagonal matrix of the known direction cosines of constrained dipole orientations and $Q^{\prime}$ is an $N$ element vector of the dipole magnitudes.

$$
\begin{aligned}
\underline{B} & =\boldsymbol{W D} \underline{Q}^{\prime}+\underline{N} \\
\boldsymbol{D} & =\left[\begin{array}{cccc}
\alpha_{1} & 0 & \cdots & 0 \\
\beta_{1} & 0 & 0 \\
\gamma_{1} & 0 & 0 \\
0 & \alpha_{2} & \vdots \\
0 & \beta_{2} & \vdots \\
0 & \gamma_{2} & 0 \\
\vdots & \vdots & 0 \\
0 & 0 & 0 \\
0 & 0 & \alpha_{N} \\
0 & 0 & \cdots & \beta_{N}
\end{array}\right] \\
\underline{Q}^{\prime}= & {\left[\begin{array}{ll}
\left|Q_{1}\right|,\left|Q_{2}\right|, & \cdots
\end{array} Q_{N}^{T}\right.}
\end{aligned}
$$

then (4) becomes

$$
\begin{gathered}
\underline{B}=\boldsymbol{H} \underline{Q}^{\prime}+\underline{N} \\
\boldsymbol{H}=\boldsymbol{W D}=\left[\begin{array}{ccc}
\boldsymbol{H}_{1,1} & \cdots & \boldsymbol{H}_{1, N} \\
\vdots & \vdots \\
\boldsymbol{H}_{M, 1} & \cdots & \boldsymbol{H}_{M, N}
\end{array}\right] \\
\boldsymbol{H}_{m, n}=\frac{\mu_{0}}{4 \pi\left|\underline{r}_{m}-\underline{r}_{n}\right|^{3}}\left[\begin{array}{c}
r_{z: m, n} \beta_{n}-r_{y: m, n} \gamma_{n} \\
-r_{z: m, n} \alpha_{n}+r_{x: m, n} \gamma_{n} \\
r_{y: m, n} \alpha_{n}-r_{x: m, n} \beta_{n}
\end{array}\right]
\end{gathered}
$$


Note that (5) includes the measured data in $\underline{B}$ for all three vector components of the field at each sample point. If only normal measurements are taken, then the projection of the normal field onto these rectangular vector components is used. This formulation and model permit significant reduction in the dimensionality of the problem, but in general, still do not lead to a unique solution.

\section{Resolution Limits and Solution Ambiguity}

Though NMI is, we feel, a feasible and promising technology, there are underlying physical limitations which must be considered. The resolution in our system and the ability to come to a single unique solution are limited by several factors. We will consider the following three problems.

1) The "ill-posed" nature of the system equations.

2) The resolution characteristics of the SQUID gradiometer.

3) Noise, i.e., the magnitude of the background magnetic fields compared to the neuromagnetic field.

\section{A. System Equation Considerations}

Consider the linear problem $\underline{B}=\boldsymbol{H} \underline{Q}^{\prime}+\underline{N}$ of (5) where the system matrix $\boldsymbol{H}$ is of dimension $3 M \times N$. We will investigate the properties of this matrix. We may expand $\boldsymbol{H}$ using the singular value decomposition [13]:

$$
\boldsymbol{H}=\boldsymbol{U} \boldsymbol{\Lambda}^{1 / 2} \boldsymbol{V}^{T}
$$

where $\boldsymbol{\Lambda}^{1 / 2}$ is a diagonal matrix of singular values, ranked in order of descending magnitude, equal to the square root of the eigenvalues of $\boldsymbol{H H}^{T}$ or $\boldsymbol{H}^{T} \boldsymbol{H}$. $\boldsymbol{U}$ and $\boldsymbol{V}$ are matrices with columns equal to the eigenvectors of $\boldsymbol{H H}^{T}$ and $\boldsymbol{H}^{T} \boldsymbol{H}$, respectively. From the orthogonality of the columns $\underline{U}_{i}$ and $\underline{V}_{i}$ of $\boldsymbol{U}$ and $V$ we can rewrite the expansion as [13]:

$$
\begin{aligned}
& \boldsymbol{H}=\sum_{i=1}^{R} \lambda_{i}^{1 / 2} \underline{U}_{i} \underline{V}_{i}^{T} \\
& \underline{B}=\sum_{i=1}^{R} \lambda_{i}^{1 / 2} \underline{U}_{i} \underline{V}_{i}^{T} \underline{Q}^{\prime}+\underline{N}
\end{aligned}
$$

where $R$ is the rank of $\boldsymbol{H}$, i.e., the number of nonzero eigenvalues in $\boldsymbol{H H}^{T}$ or $\boldsymbol{H}^{T} \boldsymbol{H}$. We can form the pseudoinverse $\boldsymbol{H}^{\dagger}$ of $\boldsymbol{H}$ and write the least squares minimum norm solution of (5) as

$$
\underline{Q}^{\dagger}=\sum_{i=1}^{R} \frac{1}{\lambda_{i}^{1 / 2}} \underline{V}_{i} \underline{U}_{i}^{T} \underline{B} .
$$

In (6), the component of $\underline{B}$ due to the projection of $Q^{\prime}$ through $\underline{U}_{i} \underline{V}_{i}^{T}$ is weighted by $\lambda_{i}^{1 / 2}$ thus if $\lambda_{i}^{1 / 2}$ is small, the resulting contribution to the data is small. In the pseudo inverse however, $1 / \lambda_{i}^{1 / 2}$ will be large and hence the component of the noise vector projected through $\underline{V}_{i} \underline{U}_{i}^{T}$ will be disproportionately amplified. To avoid this problem, it is common to truncate the summation in (7) to sum over $P$ $\leq R$ values so as to reduce the error in $\underline{Q}^{\dagger}$ due to measurement noise. This truncation however, produces another type of error in the solution image by reducing the resolution and fine detail available from the small singular values. An optimal truncated pseudoinverse solution is obtained when $P$ is chosen to minimize the sum of noise error plus resolution error [13]. This optimal truncation index, under the assumption that $\underline{Q}$ and $\underline{N}$ are independent, white Gaussian vectors, is given by:

$$
P_{\mathrm{opt}}=\max _{i}\left[i \mid \lambda_{i} \geq \frac{E\left\{\underline{N}^{T} \underline{N}\right\}}{E\left\{\underline{B}^{T} \underline{B}\right\}}\right] .
$$

The number of terms $P$ used in the summation of (7) determines the possible dimension of the solution. As $P$ is increased towards $R$, the dimension of the solution, and hence potential resolution, is increased, but at the cost of increased sensitivity to noise.

This development of an optimum truncated pseudoinverse solution suggests that much can be learned about the stability, attainable resolution, and dimensionality of an image solution by analyzing the singular values of the system matrix $\boldsymbol{H}$. We have analyzed $\boldsymbol{H}$ obtained from several configurations of reconstruction volume and sampling surfaces. Fig. 3(b) is a plot of the square of the ordered singular values for $\boldsymbol{H}$ obtained from a sampling surface and a reconstruction space as shown in Fig. 3(a). It can be seen that $\lambda_{i}$ drops off rapidly, that $\boldsymbol{H}$ is not of full rank, and that 90 percent of the "power" is contained in the first 10 values. Even if we include 99 percent, we need only 40 values. This implies that for a signal to noise ratio of $20 \mathrm{~dB}$, we should expect to be able to recover an image consisting of at most 40 mutually orthogonal components and hence it is not possible to resolve more than 40 independent features in the solution. Consequently, it would be unwise to attempt reconstructing more than 40 dipoles. We stress that there is no direct correspondence between the basis vectors of the SVD and a set of dipoles. However, we have attempted to give a qualitative argument concerning the limitation on the amount of information we can recover from the data. These conclusions are justified in the simulations presented in Section IV.

Analysis of different configurations for $\boldsymbol{H}$ show that increasing the number of sample points past some limit yields little improvement in resolution. Fig. 3(c) shows a plot of the squared magnitude of singular values of $\boldsymbol{H}$ for the same system as in Fig. 3(a), but with a second layer of hemispherically spaced sample points with a $6 \mathrm{~cm}$ radius. More than doubling the number of data samples in this configuration results in virtually no change in the relative magnitudes of the singular values [(compare Fig. 3(b) to 3(c)] indicating that the additional measurements contain virtually no useful information, i.e., the dimension of the solution is still restricted to about 40 basis vectors.

In another experiment, the spacing between dipole voxels was doubled, reducing the number of voxels in the reconstruction (or equivalently the number of elements in 


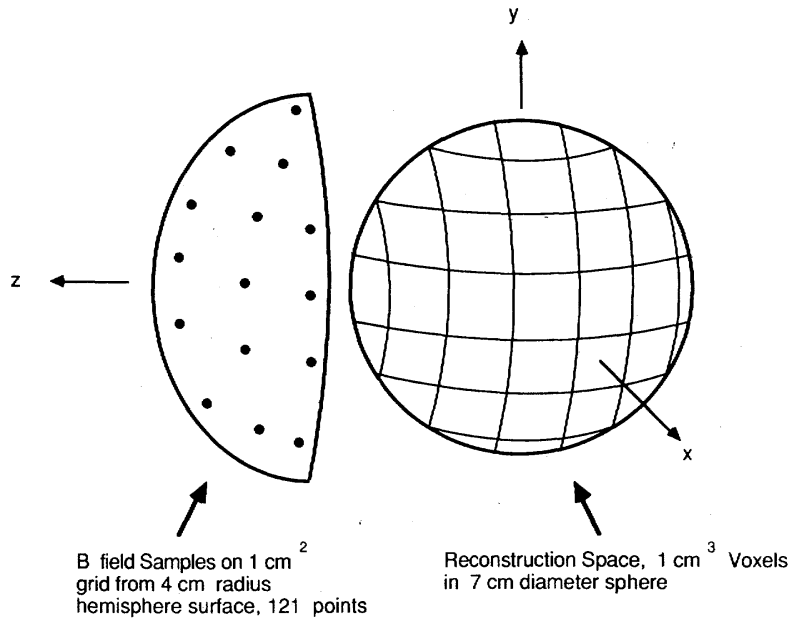

(a)

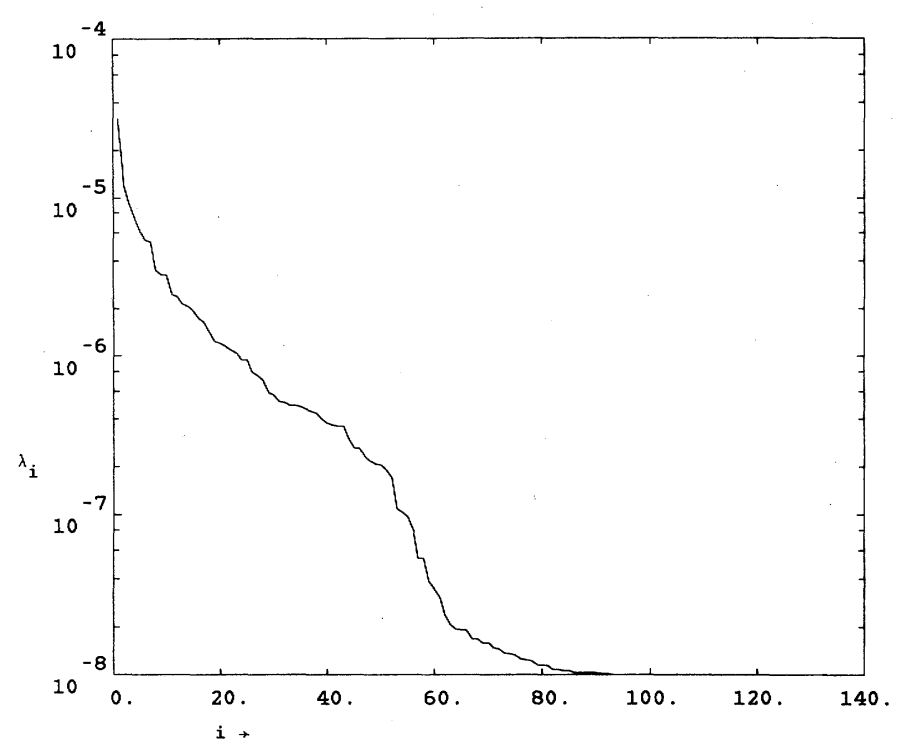

(c)

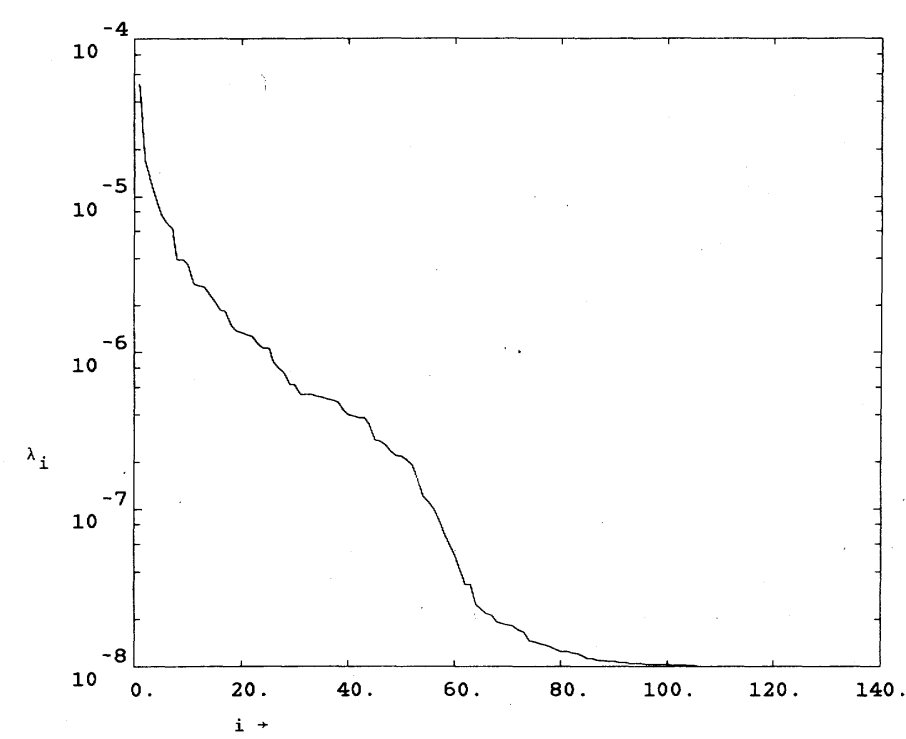

(b)

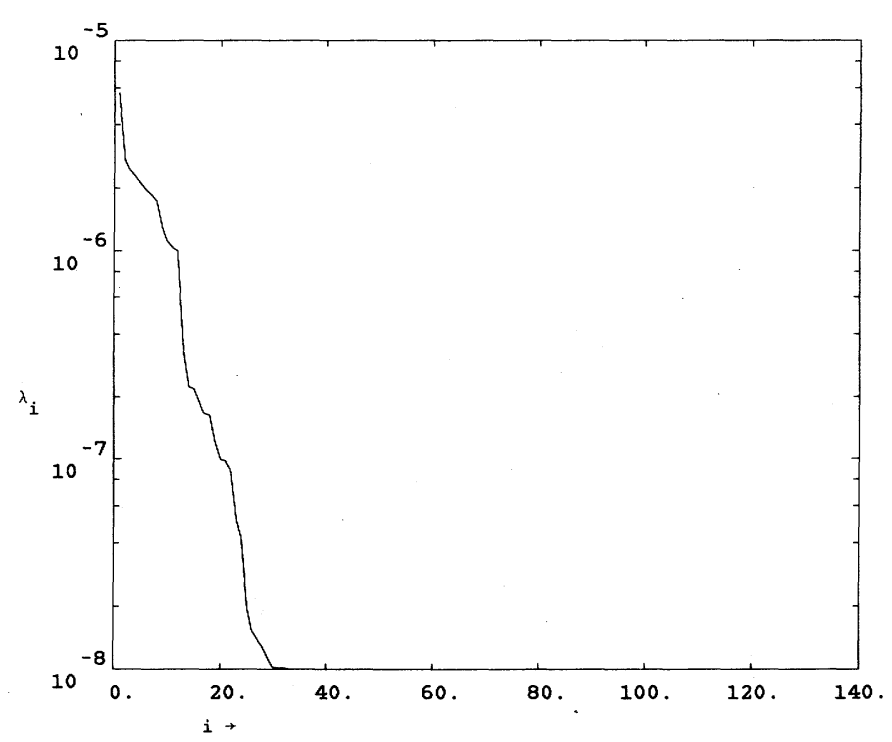

(d)

Fig. 3. (a) Geometry of reconstruction space and sample surface used for SVD analysis of system matrix. (b) Plot of squared singular values for geometry of (a). (c) Plot of squared singular values for double layer sampling hemisphere surface. (d) Plot of squared singular values for reduced resolution $\left(2 \mathrm{~cm}^{3}\right.$ voxels $)$ in reconstruction space.

$Q$ ) by a factor of eight, while the sample points of Fig. 3(b) were retained. This produced an $\boldsymbol{H}$ with singular values as shown in Fig. 3(d). Though lower resolution and fewer cells were used, the number of large singular values dropped by a factor of two as compared to the 8 to 1 drop in voxels. Thus, we can produce a less ill-conditioned system at the expense of forming a lower resolution solution.

The Biot-Savart equation describes a system which is increasingly "low-pass" to spatial frequencies as the distance from detectors increases. This is due to the vector cross product relationship between the dipole and the distance vector to the magnetic field measurement. As $\left(\underline{r}_{m}-\underline{r}_{n}\right)$ increases, the ability to resolve two distinct dipoles decreases, while at the same time the signal strength drops off as $1 / r^{2}$ until the measurement precision needed for resolution is lost in noise.

In conclusion, an exact reconstruction is not in general possible for all current distributions, even in the case where we have many more magnetic samples than dipole voxels. We have found, however, that the imposition of prior constraints on the solution enables reconstruction of certain classes of images.

\section{B. SQUID Resolution}

Present neuromagnetic research uses biomagnetometer instrumentation based on SQUID technology. A typical system consists of a set of magnetic pickup coils wound in a second-order gradiometer configuration, the SQUID 
detector, associated electronics, and a dewer to encase the coils and SQUID in liquid helium in order to keep them at superconducting temperatures. In a closed superconducting coil, the magnetic flux through the coil is constant, so any external magnetic field threading the coils causes a current to flow to oppose the external field. This current is coupled to the SQUID detector which serves as a low-noise, high-gain current to voltage amplifier. The SQUID biomagnetometer using second-order detection coils provides sufficient sensitivity and noise immunity to make neuromagnetic measurements in an unshielded laboratory environment possible. SQUID systems are commercially available in several configurations including single channel and five or seven channel array devices.

There are several practical limitations to the system spatial resolution which are introduced by the SQUID biomagnetometer. We will discuss three of these: pickup coil size, gradiometer sensitivity as a function of distance, and data acquisition time.

The SQUID pickup coil used in our experiments is representative of those common in neuromagnetic research and is $2.0 \mathrm{~cm}$ in diameter. The magnetic flux at a point is estimated by the average flux through this pickup coil, and its diameter can have a significant effect on resolution [14]. This effect is most evident at close ranges as shown in Fig. 4 which plots the gradiometer response compared to the point flux density from a single tangential dipole at a depth of $0.25 \mathrm{~cm}$ as the dipole is moved tangentially across the SQUID's field of view. The smoothed curve from the gradiometer can be interpreted as its spatial impulse response to a point dipole source. Deconvolution of this impulse response in the data can restore the original resolution, but in the presence of measurement noise, this approach is limited and the recoverable resolution is related to the coil diameter. We have chosen a $1 \mathrm{~cm}$ cube voxel size which agrees with Okada's [5] finding that dipoles separated by $1-2 \mathrm{~cm}$ are resolvable.

The second-order spatial gradiometer configuration of the SQUID pickup coils is used to suppress large uniform fields such as the earth's magnetic field. It is this arrangement which allows the collection of data in a nonshielded environment. As stated above, the measured field strength drops off as the inverse square of the distance to the source, however the gradiometer increases the rate of this attenuation resulting in poor detection of the field due to dipoles located at larger distances from the detector.

Figs. 5 and 6 demonstrate the attenuation as a function of distance due to the second-order gradiometer configuration. At $2 \mathrm{~cm}$ depth, the response is a fair approximation to the point flux density. Moving from $2 \mathrm{~cm}$ to $16 \mathrm{~cm}$ depth causes the response to drop $10 \mathrm{~dB}$ more than predicted from the inverse square reduction in flux density. Also, the peaks in the response are shifted toward the center line. In the range of $2-6 \mathrm{~cm}$ depth, which is our primary range of interest for cortical activity, the gradiometer gives a reasonable approximation to the point flux through the center of the coil.

The system matrix $\boldsymbol{W}$ of (3) should be modified to ac-

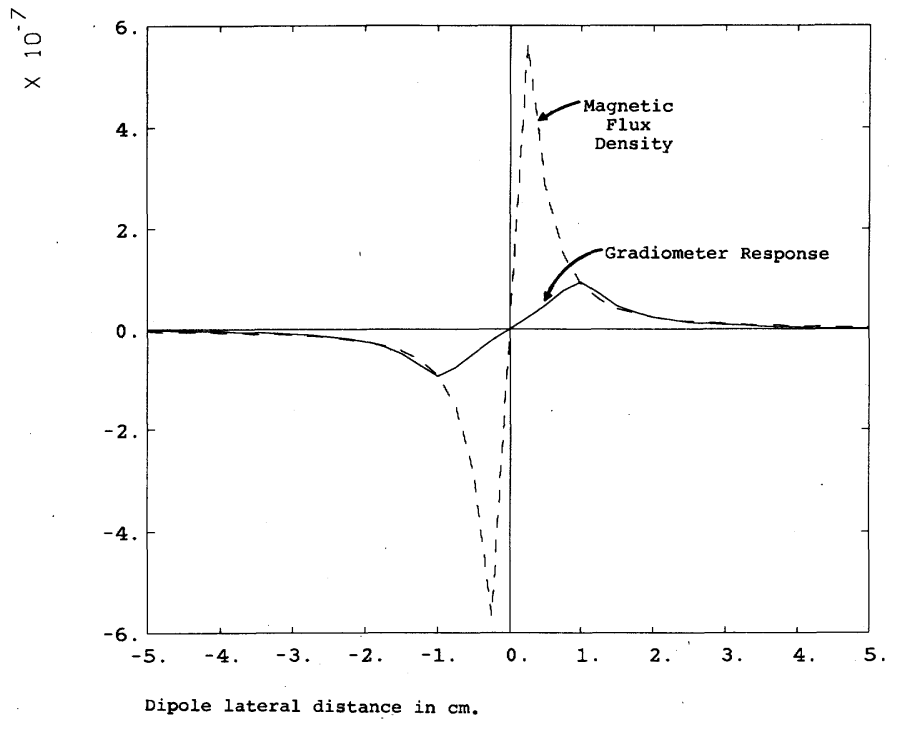

Fig. 4. Gradiometer response and flux density versus lateral position for a single current dipole at $0.25 \mathrm{~cm}$ axial.

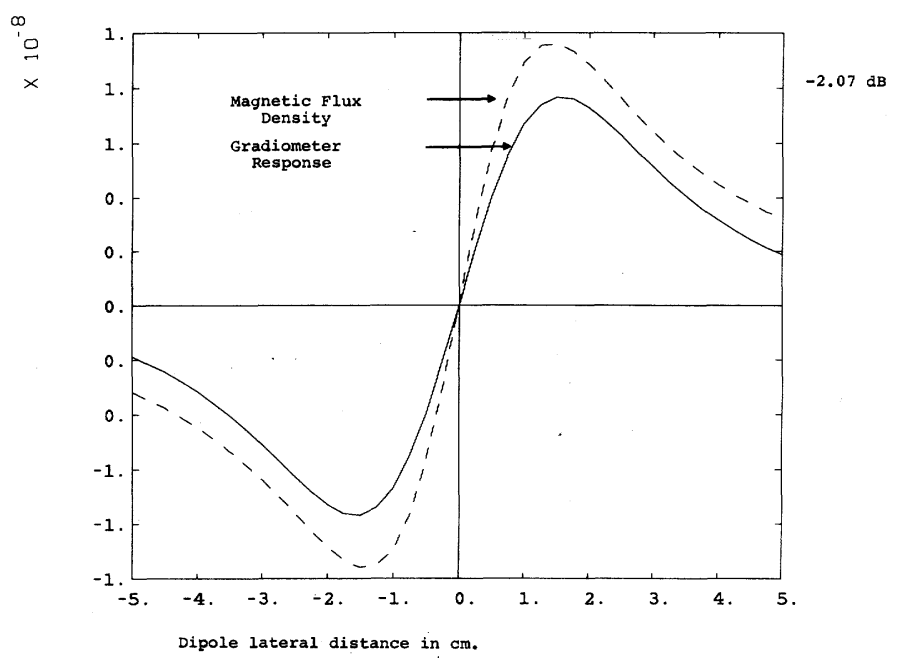

Fig. 5. Gradiometer response and flux density versus lateral position for a single current dipole at $2.0 \mathrm{~cm}$ axial depth.

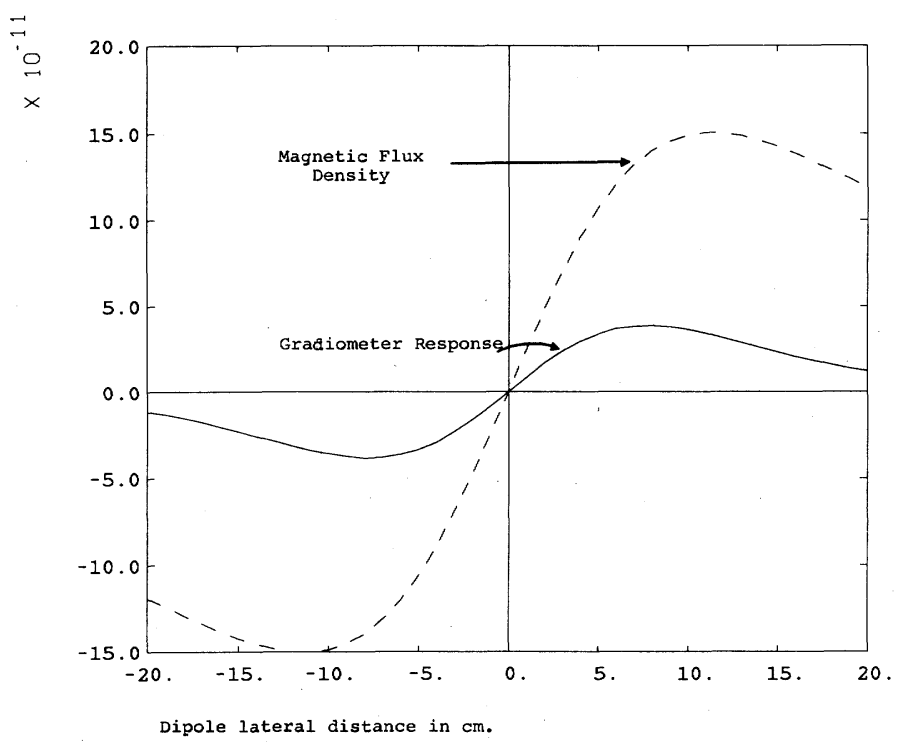

Fig. 6. Gradiometer response and flux density versus lateral position for a single current dipole at $16.0 \mathrm{~cm}$ axial depth. 
count for the effects of coil size and gradiometer configuration in order to accurately represent the physical system. A usable approximation is obtained if coil diameter is neglected and $\boldsymbol{W}$ is formed as a sum of matrices corresponding to the different coil positions in the gradiometer. In our device there are three coils with axial spacing between them of $50 \mathrm{~mm}$; the center one is counter-wound and contains twice as many turns as the other two. With this configuration, $\boldsymbol{W}$ is computed as follows:

$$
\boldsymbol{W}=\boldsymbol{W}^{1}-2 \boldsymbol{W}^{2}+\boldsymbol{W}^{3} \text {. }
$$

where $\boldsymbol{W}^{1}, \boldsymbol{W}^{2}$, and $\boldsymbol{W}^{3}$ are matrices computed separately as in (3) but using the centers of the three coils respectively when computing the position vectors $\underline{r}_{m}$. A more accurate expression can be found by integrating the matrix form of the Biot-Savart equation found in (3) over the cross section area of each coil, i.e.,

$$
\boldsymbol{W}_{m, n}^{1}=\int_{\phi_{\mathrm{m}}^{1}} \boldsymbol{W}_{m, n} d \underline{r}_{m}
$$

where $\phi_{m}^{1}$, the integration surface, corresponds to the disk enclosed by the front pickup coil. This method was used in obtaining the plots of Figs. 4, 5, and 6. We have found, however, that as suggested by Fig. 5, at the depth range corresponding to the cortex, the simple point measurement model of (3) is sufficient and using (9) or (10) produces little noticeable improvement in the reconstructed image.

The logistics of data acquisition also impose a practical limit on the number of data points which can be gathered. Functional neuromagnetic fields of the brain are weak enough that it is essential to perform time averaging between successive sets of data for noise suppression. For measurements from evoked responses, ten or more sample windows, each approximately one second long and synchronized to the patient stimulus, are needed at each sample point. With a single channel SQUID, this acquisition time and the time required to reposition the SQUID detector imply that data from a small $17 \times 17$ cell grid could take on the order of hours to gather. The seven channel array devices are an improvement, but we look to large array of SQUID detectors in the future to eliminate this restriction on resolution.

\section{Noise and Background Magnetic Fields}

The functional neuromagnetic fields of the brain which are of interest in neuromagnetic imaging are on the low end of the detectable scale. Somatically, visually, and auditory evoked fields have been measured at levels near 0.1 pico Tesla [8]. It is presumed that fields generated by higher level thought processes would have an even smaller field strength. This can be compared to magnetic fields from the alpha and delta rhythms which are at $1 \mathrm{pT}$, the steady field of the eye at $10 \mathrm{pT}$, and the magnetocardiogram at 40-50 pT [8]. Many of these biologically generated fields are present and larger than the field of interest in neuromagnetic measurements and so must be filtered out before usable results can be obtained.
The self noise spectral level of our SQUID detector is specified to be below $20 \mathrm{f} \mathrm{T} / \mathrm{Hz}^{1 / 2}$. This is sufficiently low to permit detection of the fields of interest, thus the limiting factor is the noise from background magnetic fields. The earth's steady-state magnetic field has an amplitude at 40 degrees latitude of approximately $50 \mu \mathrm{T}$ and interference from commercial power can reach $100 \mathrm{nT}$ [15]. Nearby feromagnetic objects can introduce gradients into these fields and nearby machinery can cause additional interference. The maximum noise spectral level above $1 \mathrm{~Hz}$ can range from $10 \mathrm{f} \mathrm{T} / \mathrm{Hz}^{1 / 2}$ for magnetically shielded rooms to $10 \mathrm{nT} / \mathrm{Hz}^{1 / 2}$ for noisier hospital and laboratory locations [15]. The second-order gradiometer configuration of the pickup coils can attenuate the zeroand first-order gradients of these fields to acceptable levels, but care must still be taken to reduce local interference. This background noise level will set a limit on the system sensitivity, and thus on the achievable resolution when dealing with the weak signals of interest. Even with the apparently overwhelming problems with noise, researchers have successfully made neuromagnetic measurements of brain signals in common laboratory environments [1], [2], [3], however the additional precision required for high-resolution imaging makes the use of shielded rooms and a large array of sensors, for reduced data acquisition time, the most attractive arrangement for NMI.

\section{Estimation Algorithms}

There are several well known algorithms used in image reconstruction problems where solutions to ill-posed underdetermined linear systems are required [16]. These algorithms use a cost criterion to select a single solution from the infinite possible solutions to $\underline{B}=\boldsymbol{H} \underline{Q}$.

In the following discussion of algorithms we assume noiseless data. Algorithm performance with noisy data is discussed in Section IV-C. Sections IV-A and -B. present the algorithms and their implementations, the results of the simulated comparisons are presented in Section IV-C.

\section{A. N-Dipole Solutions}

Given the data vector $\underline{B}$ and assuming there is no noise, then a single solution can be obtained by solving the general problem

$$
\min _{Q \in R^{N}}\left[\begin{array}{ll}
g(\underline{Q}) & \underline{B}=H \underline{Q} \\
\text { subject to } & \underline{Q} \geq 0
\end{array}\right]
$$

where $g(\underline{Q})$ denotes some functional on the solution vector. The choice of the cost function will determine the class of solutions.

The most common cost function is the minimum norm solution, with $g(\underline{Q})=\underline{Q}^{T} \underline{Q}$, which can be found by numerous pseudoinversion techniques. We have used the algebraic reconstruction technique (ART) which is an iterative algorithm popular in medical imaging that converges to the minimum norm solution [16]. 
The algorithm steps are as follows: Let $\underline{H}_{i}^{T}$ be the $i_{\text {th }}$ row of $\boldsymbol{H}$, then for iteration step $k$ :

$$
\begin{aligned}
\underline{Q}^{k+1} & =\underline{Q}^{k}+\frac{B_{i}-\underline{H}_{i}^{T} \underline{Q}^{k}}{\underline{H}_{i} \underline{H}_{i}^{T}} \underline{H}_{i} \\
\text { for } k & =0,1, \cdots k_{e}, \quad i=k_{\bmod M}+1 \\
e_{k} & =\frac{\left|\underline{B}-H \underline{Q}^{k}\right|^{2}}{|\underline{B}|^{2}} .
\end{aligned}
$$

Iterations terminate at $k_{e}$ when the error $e_{k}$ drops below a predetermined limit. The minimum norm approach favors smooth solutions; we have found it will tend to force the solution dipoles as close as possible to the SQUID detectors since the field falls off as the inverse square of the distance between the detector and source. $g(\underline{Q})$ is minimized when smaller current dipole magnitudes are located near the detectors to yield equivalent magnetic field measurements. The bias toward a solution near the detector and the underdetermined nature of the system make depth resolution difficult with a straightforward minimum norm solution. This problem may be overcome by forming a weighted minimum norm solution, $g(\underline{Q})=\underline{Q}^{T} C^{T} \boldsymbol{C} \underline{Q}$ where $C$ is a weighting matrix which compensates for the inverse square bias. Our experiments, however, show that a workable value for $\boldsymbol{C}$ is difficult to predict theoretically and is highly dependent on the detailed measurement geometry. Even though depth bias may be removed with proper selection of $\boldsymbol{C}$, the reconstructed images are very smooth tending to smear single dipole sources over the entire 3-D reconstruction volume.

An alternative formulation was proposed by Dallas [8] based on Maxwells' equations

$$
\nabla \times B=\mu_{0} J, \nabla \cdot B=0 .
$$

Taking the Fourier transforms of (11) yields a set of linear equations relating the current and magnetic fields. By sampling the Fourier transform of the two fields and decomposing the magnetic field into two regions, the measurement region and a "forbidden region"' over which the field cannot be measured, a large set of linear equations can be formed. The unknowns in the equation are the samples of the Fourier transforms of the current field and the magnetic field in the forbidden region. In the algorithm, the reconstruction volume and measurement region are discretized into sample cells as in our model. This formulation has the advantage that it provides simultaneous reconstruction of the internal current and magnetic fields. Dallas has demonstrated successful two-dimensional reconstructions of simulated data. The Fourier space solution approach, however, does not eliminate the ill-posed nature of the problem or reduce the null space of the system matrix $\boldsymbol{H}$ which leads to the infinite set of possible solutions. Given measurements $\underline{B}$, any solution to (3) is also a solution to (11). The reconstructed images using the Fourier domain approach appear to be of minimum norm type. This implies that problems of smearing and bias toward detector surfaces would also arise here with three-dimensional reconstructions.

Another technique is to produce the maximum entropy solution where we minimize the functional

$$
\begin{aligned}
g(\underline{Q})=\sum_{i=1}^{N} \frac{Q_{i}}{\|\underline{Q}\|_{L 1}} \operatorname{Ln} \frac{Q_{i}}{\|\underline{Q}\|_{L 1}}, \\
Q_{i} \geq 0,\|\underline{Q}\|_{L 1}=\sum_{i=1}^{N} Q_{i} .
\end{aligned}
$$

This technique is favored by many researchers [18], [19] as it yields the maximally uniform image consistent with the data.

There are a number of algorithms for maximum entropy restoration. Most require the elements of $\boldsymbol{H}$ to be nonnegative [16], [20], which does not meet our needs. Nonnormalized algorithms are unacceptable due to the nonlinear effect on the solution caused by scaling the data. We have used an algorithm based on techniques described in [20] for a normalized maximum entropy reconstruction with a general system matrix $\boldsymbol{H}$. The iterative steps are as follows:

$$
\begin{aligned}
& \underline{Q}^{0}=[1.0,1.0, \cdots]^{T} \\
& Q_{j}^{k+1}=Q_{j}^{k} \exp \left[\omega\left(H^{T} \underline{\lambda}^{k}\right)_{j}\right] \\
& \underline{\lambda}^{k}=\frac{\left(\underline{B}-H \underline{Q}^{k}\right)}{\|\underline{Q}\|_{L 1}} \\
& \quad \text { for } k=0,1, \cdots, k_{e}, \quad i=k_{\bmod N}+1 .
\end{aligned}
$$

$\omega$ is a relaxation constant to control convergence. As above, iterations are terminated when $e_{k}$ drops below an acceptable limit. The entropy expression is defined only for $Q_{i}^{k} \geq 0$, so we are required to use our dipole orientation constraint model (5) so that $\underline{Q}$ becomes a vector of nonnegative magnitudes. This maximum entropy approach has shown in our simulation experiments to suffer from the same bias toward near detector solutions as the minimum norm image.

\section{B. Minimum Dipole Solutions}

An alternative class of solutions can be found by attempting to minimize the number of dipoles in the solution. This may be written as:

$$
\min _{P \leq \operatorname{Rank}(H)}\left[\min _{S^{P} \subset R^{N}}\left[\min _{Q \in S^{P}}\left\|\underline{B}-H_{S} \underline{Q}\right\| \text { such that } \underline{Q} \geq \underline{0}\right]\right]
$$

where $H_{S}$ is an $M \times P$ matrix relating to a set of $P$ dipoles from $S^{P}$, a $P$-dimensional subspace of $R^{N}$, and $N$ is the total number of dipole cells. A solution to this expression gives the minimum norm solution which simultaneously minimizes the number $P$ of nonzero dipoles over all possible combinations of dipoles. This formulation is appealing since the system is underdetermined and thus choosing the minimum number of dipoles is maximally noncomittal in the sense that unless we have good reason to believe otherwise, the minimum-order solution is the 
least activity which could have given rise to the data. If the minimum dipole representation is a reasonable model of the actual current distribution, then this approach also enables resolving current dipoles in depth by removing the underdetermination in the system.

The disadvantage of this approach is that the optimization problem is nondifferentiable, thus conventional gradient search algorithms cannot be employed. Initial simulations were performed using an exhaustive search as follows:

1) Order $P=1$.

2) Find least squares solutions for all possible combinations of $P$ dipoles; choose solution with least error.

3) If error is greater than predetermined confidence limit, then $P=P+1$.

4) Repeat steps 2) and 3) as necessary.

This approach is impractical for anything but small reconstruction spaces, but provides excellent resolution laterally and in depth. Some speed improvement has been obtained by using directed or pattern search techniques [21] to structure the search to select combinations of nonzero dipoles which progressively reduce the error. From a random starting position dipoles are moved step-wise only in directions which decrease error until a stable position is found. The number of dipoles $P$ is then increased, and for a new search, until increasing the number of dipoles does not lead to reduced error.

\section{Comparison of Algorithm Performance}

1) Noiseless Data Reconstructions: Simulated magnetic field point measurements were used in a comparison of the algorithms discussed above. Three disjoint current dipoles of differing magnitude within a sphere were modeled, and noiseless measurements on a hemispherical surface surrounding them were computed. The sphere was 3 $\mathrm{cm}$ in radius centered at $(0,0,0)$ and divided into $1 \mathrm{~cm}^{3}$ voxels. The simulated magnetic samples were taken on a hemisphere of radius $4 \mathrm{~cm}$ with $z \geq 0$. In reconstruction, all dipoles were constrained to be in the $+x$ direction. The three dipoles were located at $(x, y, z)$ coordinates $(1,-1$, $1),(1,-1,-2)$, and $(1,2,0)$ with magnitudes $1.0,1.5$, and 2.0 , respectively. This set of sources was chosen to demonstrate problems associated with the minimum norm and maximum entropy solutions, i.e., their inability to resolve depth from the measurement surface.

A single plane $(x=1)$ of the reconstructed 3-D images from the ART, maximum entropy, and minimum dipole algorithms are shown in Fig. 7. The magnetic sample hemisphere surface surrounds the left half of the images. Since all three sources are located in the $x=1$ plane, this allows a comparison of the depth resolution for each algorithm. It can be seen that the minimum norm and the maximum entropy images shift energy toward the sphere surface and blur the dipole locations. The maximum entropy entirely misses the deeper lying dipoles. The ART image produced a smoothed cluster of intensity near the $(1,2,0)$ dipole, but the maximum voxel, not shown, is at $(2,2,0)$ which is near the sphere edge. The maximum

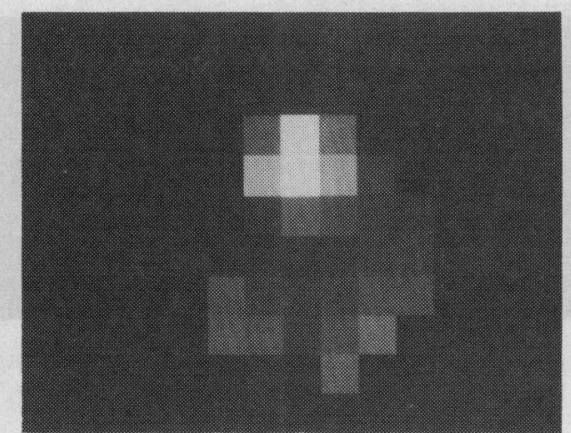

(a)

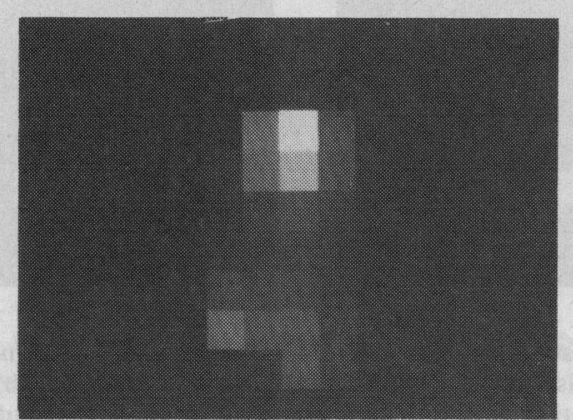

(b)

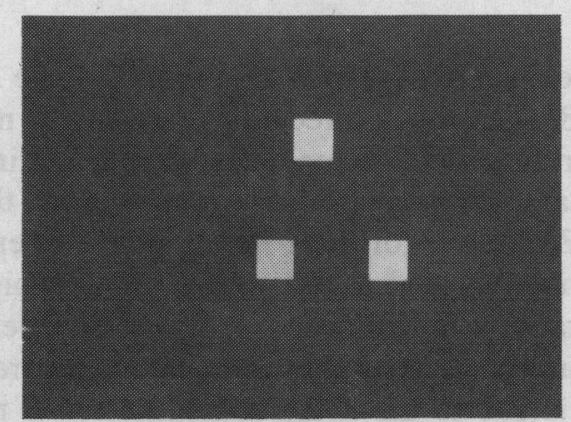

(c)

Fig. 7. Noiseless image reconstruction for a three dipole source. $x=1$ plane shown. Intensity is $+x$ component of dipole field; (a) ART (minimum norm) solution, (b) maximum entropy solution, and (c) exhaustive search minimum dipole solution.

entropy approach produced a slightly less disperse solution, but still had a smoothed cluster around $(1,3,0)$. It did, however, produce a more uniform field of near zero values away from this cluster than did the ART algorithm. The minimum dipole search algorithm produced the correct solution. The number of dipoles, their depth, and relative positions were estimated exactly, and their magnitudes were accurate to three decimal places. It is important to note that each of these three images represents an accurate solution vector $\underline{Q}$ to $\underline{B}=\boldsymbol{H} \underline{Q}$ for the measured data. The fact that they differ so dramatically confirms the need to select the model and algorithm best suited to our current knowledge of the physical processes involved in neural activity.

2) Reconstruction with Noisy Data: The experiment was repeated with independent Gaussian noise added to the magnetic measurement vector at a $20 \mathrm{~dB}$ signal to noise ratio. Fig. 8(a) and (b) shows the results of ART and Max. Entropy reconstructions, respectively. Fig. 8(c) 


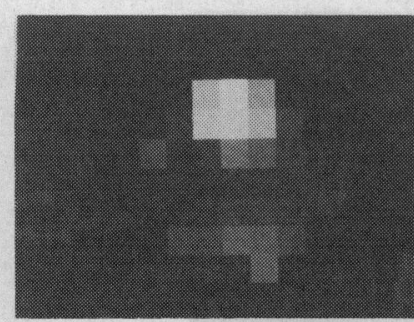

(a)

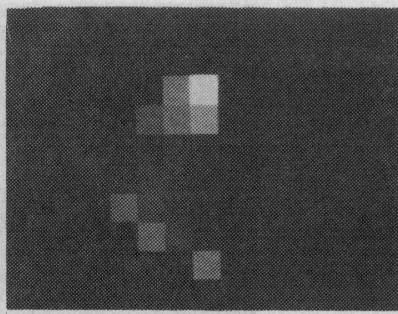

(b)

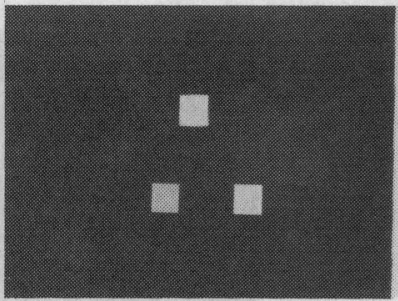

(d)
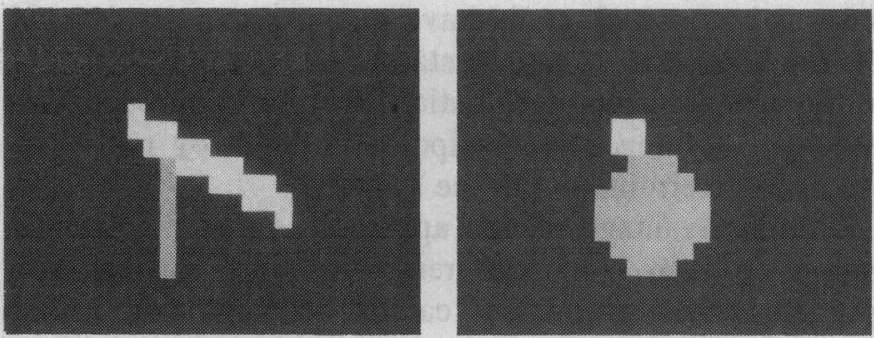

(a)
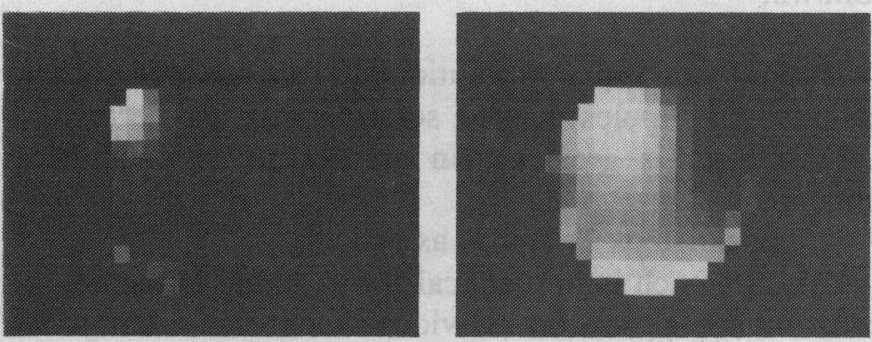

(b)
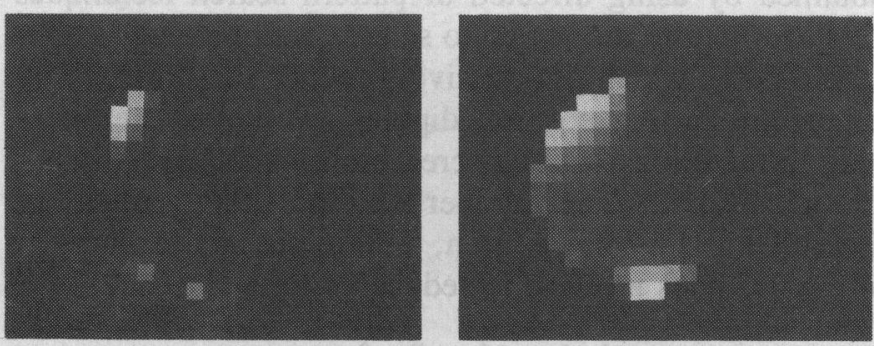

(c)

Fig. 9. Reconstruction of bar and disk source in a $13 \mathrm{~cm}$ diameter sphere, (a) $x=-1$ (left) and $Z=2$ (right) planes of source distribution, (b) ART reconstruction, and (c) maximum entropy reconstruction.

these data. The effectiveness of the minimum dipoles solution for the simple three dipole source cannot guarantee that larger distributions like this would produce similarly successful images. A complex source would not be correctly reconstructed as a minimum dipole image if a simpler equivalent to the source exists (in the sense of producing the same magnetic measurements). It can be argued though, that without prior knowledge it is unreasonable to select a more complex reconstruction (more dipoles) over a simple one. It is, however, clear from Fig. 9 that the common minimum norm or maximum entropy methods result in unacceptable solutions. This conclusion motivates our continued effort to develop more efficient algorithms to compute higher order minimum dipole reconstructions.

\section{Conclusions}

We have investigated some of the fundamental limitations on the resolution which may be achieved in a neuromagnetic imaging system. While it has been demonstrated that neuromagnetic data may be used to accurately locate the position of a single current dipole implanted in a human skull [1], [4], [5], it is clear from the results presented in Sections II and III that the resolution of the system for distributed sources is severely limited by the 
ill-posed nature of (5). It therefore appears unlikely that high resolution volume images of brain activity, comparable to images obtained from an emission tomography system for example, can be reconstructed using SQUIDbased magnetic measurements. However, the use of restricted models, such as those used by Singh et al. [7] or those presented in Section II-B, in conjunction with a minimum dipole approach should lead to clinically useful results. From the results obtained using the minimum norm and maximum entropy methods, we conclude that these approaches are not suitable for neuromagnetic imaging if the sources are actually distributed in three-dimensions.

Further work is needed to develop an efficient algorithm which can produce a minimum dipole image for larger reconstruction spaces and more dipole sources. One aspect of the problem currently under investigation is the use of statistical models for the dipole activity with parameters estimated using standard techniques from estimation theory. This approach allows us to specifically include the presence of noise in the data and should also lead to computationally tractable solutions to the minimum dipole problem.

\section{REFERENCES}

[1] Y. Okada, Biomagnetism: An Interdisciplinary Approach. New York: S. J. Williamson, G. L. Ramani, L. Kaufman and I. Modena, Eds. New York: Plenum, 1982, pp. 399-482.

[2] D. Brenner, S. J. Williamson, and L. Kaufman, "Visually evoked magnetic fields of the human brain," Science, vol. 190, pp. 480-482, 1975.

[3] D. Brenner, J. Lipton, L. Kaufman, and S. J. Williamson, "Somatically evoked magnetic fields of the human brain,"' Science, vol. 199, pp. 81-83, 1978.

[4] B. N. Cuffin, "A comparison of moving dipole inverse solutions using EEG's and MEG's,' IEEE Trans. Biomed. Eng., vol. BME-32, pp. 905-910, 1985.

[5] Y. Okada, "Discrimination of localized and distributed current dipole sources and localized single and multiple sources," in Biomagnetism: Applications and Theory, H. Weinberg, G. Stroink, and T. Katila, Eds. New York: Pergamon, 1985, pp. 266-272.

[6] H. Weinberg, P. Brickett, F. Coolsma, and M. Baff, "Topography of simulated MEG and EEG generated by multiple intracranial dipoles," in Biomagnetism: Applications and Theory, H. Weinberg, G. Stroink, and T. Katila, Eds. New York: Pergamon, 1985, pp. $273-$ 277.

[7] M. Singh, D. Doria, V. W. Henderson, G. C. Huth, and J. Beatty, "Reconstruction of images from neuromagnetic fields," IEEE Trans. Nucl. Sci., vol. NS-31, pp. 585-589, 1984.

[8] S. J. Williamson and L. Kaufman, "Biomagnetism," J. Magnetism and Magnetic Mater., vol. 22, pp. 129-201, 1981.

[9] D. Cohen and H. Hosaka, "Magnetic field produced by a current dipole," J. Electrocardiol., vol. 9, pp. 409-417, 1976.

[10] D. S. Barth, W. Sutherling, J. Broffman, and J. Beatty, "Magnetic localization of a dipolar current source implanted in a sphere and a human cranium,' Electroenceph. clin. Neurophysiol., vol. 63, pp. 260-273, 1986.

[11] P. L. Nunez, Electric Fields of the Brain. New York: Oxford, 1981.

[12] C. Bohm and T. Greitz, "The use of a computerized brain atlas in positrom emission tomography," poster session of Int. Workshop Phys. Eng. Comput. Multidimensional Imag. Process., sponsored by Univ. of California, Irvine, Newport Beach, CA, Feb. 1986.

[13] Y. S. Shim, Z. H. Cho, "SVD pseudoinversion image reconstruction," IEEE Trans. Acoust., Speech, Signal Processing, vol. ASSP29 , no. 4, pp. 904-909, 1981.

[14] B. N. Cuffin and D. Cohen, "Effects of detector coil size and configuration on measurements of the magnetoencephalogram," J. Appl. Phys., vol. 54, pp. 3589-3594, 1983.
[15] P. Carelli, I. Modena, and G. L. Romani, "Detection coils," in Biomagnetism: An Interdisciplinary Approach, S. J. Williamson, G. L. Ramani, L. Kaufman and I. Modena, Eds. New York: Plenum, 1982, pp. 85-99.

[16] Y. Censor, "Finite series-expansion reconstruction methods," Proc. IEEE, vol. 71, pp. 409-418, 1983.

[17] W. J. Dallas, "Fourier space solution to the magnetostatic imaging problem," Appl. Opt., vol. 24, pp. 4543-4546, 1985

[18] S. F. Burch, S. F. Gull, and J. Skilling, "Image restoration by a powerful maximum entropy method," Comput. Vis., Graph., Image Processing, vol. 23, pp. 113-128, 1983.

[19] B. R. Frieden, "Restoring with maximum likelihood and maximum entropy," J. Opt. Soc. Amer., vol. 62, pp. 511-518, 1972.

[20] T. Elfving, "On some methods for entropy maximization and matrix scaling,"'Linear Algebra and its Applications, vol. 34, pp. 321-339, 1980.

[21] R. Hooke and T. A. Jeeves, "Direct search solution of numerical and statistical problems, ' J. Assoc. Comput. Mach., vol. 8, pp. 212-229, 1961.

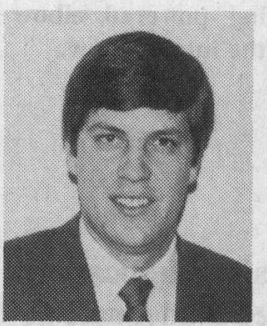

Brian Jeffs was born at Fort Meade, MD, in 1954. $\mathrm{He}$ received the B.Sc. and M.Sc. degrees in electrical engineering from Brigham Young University, Provo, UT, in 1978 and 1982 , respectively. $\mathrm{He}$ is currently working toward the Ph.D. degree from the University of Southern California, Los Angeles, in the Department of Electrical Engineering and is associated with its Signal and Image Processing Institute.

From 1977 to 1982 , he worked with Eyring Research Institute, Provo, as a Computer Systems Engineer and from 1982 to the present has been with Hughes Aircraft Company Ground Systems Group as a Sonar Signal Processing Staff Engineer. Areas of interest and research include medical image processing, threedimensional image reconstruction, and sonar signal processing.

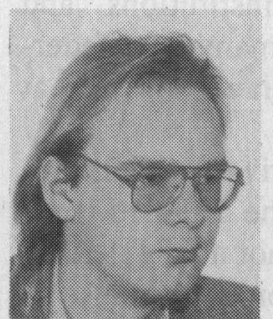

Richard Leahy (M'86) was born in Surrey, England, in 1960. He received the B.Sc. and Ph.D. degrees in electrical and electronic engineering from the University of Newcastle, Tyne, England, in 1981 and 1984, respectively

In 1985 he joined the University of Southern California, Los Angeles, as an Assistant Professor in the Department of Electrical EngineeringSystems where he is a member of the Signal and Image Processing Institute. His research interests include computerized tomography, nuclear medicine, biomedical signal processing, and numerical techniques for solving inverse problems.

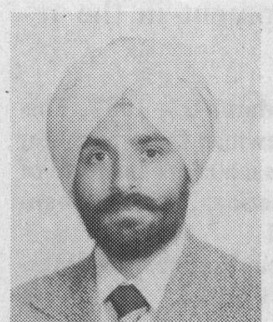

Manbir Singh (M'83) was born on October 7, 1945 in Amritsar, India. He received the Ph.D. degree in physics from the University of California, Los Angeles, in 1971.

$\mathrm{He}$ is currently an Associate Professor of Research Radiology at the University of Southern California, Los Angeles. He has been active in research pertinent to instrumentation and image reconstruction algorithm development in medical imaging including nuclear medicine, nuclear magnetic resonance, and neuromagnetic imaging.

Dr. Singh is the nationally elected IEEE AdCom representative in nuclear medical sciences for the 1986-1989 term. 